\title{
THE RELATION BETWEEN THE ELECTROENCEPHALOGRAM AND MUSCLE ACTION POTENTIALS IN CERTAIN CONVULSIVE STATES
}

\author{
BY \\ G. D. DAWSON \\ The David Lewis Epileptic Colony and Neurological Research Unit, National Hospital, Queen Square
}

(Received 25Th February, 1946)

\section{Introduction}

STUDY in man of the relation between the occurrences in the electroencephalogram (EEG) and the muscular jerkings in convulsive states has been limited. Possibly this has been due to the difficulty of anticipating the time of onset of seizures sufficiently often, and with sufficient accuracy. In two types of case this difficulty may be overcome. The first is that in which myoclonic jerkings occur over a period of one or two hours as a prodrome of a major convulsive seizure and the second that in which a wave and spike discharge in the EEG lasts for 24 to 48 hours and is accompanied by muscular jerkings. It is the purpose of this paper to describe the results of repeated examinations of patients with these conditions.

Gibbs, Davis and Lennox (1935) showed that in those patients with epilepsy in whom a discharge of wave and spike form appeared in the EEG during a seizure, minor muscular jerkings might occur at the same rate as that of the repetition of the wave and spike complex. They also reported that in the clonic stage of a major convulsive seizure the jerkings of the muscles occurred in time either with slow waves or groups of spikes in the EEG. In neither case was fuller analysis of the temporal relations of the EEG waves and the muscular movements described. Jasper and Andrews (1938) by recording the EEG, muscle action potentials and movements studied the relations between wave and spike discharges in the EEG and the muscular jerkings during minor epileptic seizures. They found that when wave and spike discharges in the EEG were provoked by overbreathing, a burst of muscle action potentials was often associated with each wave and spike complex. In some subjects this correspondence did not occur and from this they concluded that in certain circumstances the control of the motor functions concerned might be taken over by subcortical centres. Recording of the EEG and muscle action potentials has been used by Strauss and Landis (1938) to compare the features of spontaneous convulsions and those pro- duced by cardiazol. They used the method to time the contractions of flexor and extensor muscle groups but they give few details of their results in this connection. During the severe muscular jerkings which occur in myoclonic epilepsy Grinker, Serota and Stein (1938), who studied a group of patients with a hereditary form of this complaint, were able to show that a close correspondence existed during the jerkings between groups of spikelike discharges in the EEG and the muscle action potentials. They recorded the muscle action potentials only from the biceps muscle of the arm and no simultaneous recordings from antagonistic muscle groups were reported. Their records would not allow accurate measurements of the time relations of the individual spikes of a group and any particular phase of the muscular jerkings. Confirmation of the findings of Grinker et al. is given by Jasper (1941) and Gibbs and Gibbs (1941) who also describe groups of spike-like discharges in the EEG in time with the myoclonic jerkings, but in neither case are any facts added about the time relations of the spikes and the jerks.

The present investigations have been made on two types of case with myoclonic symptoms during epileptic states. The results of simultaneous recordings during myoclonic attacks of the EEG and muscle action potentials in flexor and extensor muscle groups of the limbs will be described.

\section{Material}

The subjects studied have been selected from amongst the 450 residents at the David Lewis Epileptic Colony. In this number of habitual epileptics five patients have been found in whom severe myoclonic jerkings are a prominent symptom and one patient in whom mild myoclonic twitching in the limbs accompanies a discharge of wave and spike form in the EEG that may last up to 48 hours. Of the five patients with severe myoclonic jerkings it has been possible to predict the onset of seizures to within a few hours in two patients (337 and 407) and these have been studied repeatedly. The length of the seizure in the patient with the wave and 
spike discharge makes recording easy after the seizure has started and in this subject (377) also repeated examinations have been made.

The two patients with severe myoclonic jerkings (337 and 407) are strictly comparable as regards symptoms with those described by Grinker et al., except that they have no history suggesting a familial incidence of the complaint, and that their condition is either stationary or only slowly progressive in its severity. These patients suffer from jerkings of the muscles which are sufficiently powerful, when they occur in the legs, to cause the subject to fall heavily to the ground; or, when they occur in the arms, to cause the subject, if he is holding some quite heavy object, to throw this over his head. In neither of the patients are the jerks, which are extremely brief, associated with detectable changes in consciousness. Usually the jerks occur at progressively shorter intervals over a period of 1 to 2 hours until finally one of them passes with little pause into the tonic stage of a major convulsive seizure. Occasionally after becoming more frequent the jerks occur less often and then cease. When this happens, if the patient goes to sleep before the jerks have disappeared, on his reawakening they may reappear, increase in frequency as before, and terminate in the usual major seizure. Any excitement or attempted exertion tends to aggravate the jerkings and may precipitate the terminal seizure. Therefore, if an adequate time for examination is to be gained, the subject must relax as completely as possible when the jerkings begin. Although the period between the onset of the jerks and the final seizure in this type of case is rarely more than 2 hours, it has been possible with the excellent co-operation of the subjects and their friends in the Colony, who helped the subject to the laboratory immediately the jerks began, to examine these two patients repeatedly for periods of 1 to $1 \frac{1}{2}$ hours before their major seizure.

The third patient (377) who has been examined repeatedly is one who, besides having major convulsive seizures, is subject to attacks of mild muscular jerkings which last for a period of up to 48 hours and are associated with a continuous wave and spike discharge in the EEG. At the beginning of the attack there is slight clouding of consciousness and the twitchings of his muscles are diffuse and irregular. After about twelve hours the clouding of consciousness disappears and the jerkings become synchronous in all parts of the body and regular at about three per second. If untreated this state may continue for a further 24 hours and then the jerkings and the wave and spike discharge will cease spontaneously and suddenly. For several days before a spontaneous attack, attacks of jerking may be provoked by overbreathing. For several days after a spontaneous attack the same amount of overbreathing, or a greater anount, will produce no outburst. The jerkings of this patient have none of the violent nature of those occurring in the type of case described above and do not monopolise the entire voluntary power of the muscles concerned. Once the initial stage of diffuse twitching has passed off and the jerkings have become regular, the subject is able to walk around, talk, and dress himself, though all these activities have the jerkings superimposed on them.

\section{Methods}

Records of both the EEG and muscle action potentials have been made with inkwriting oscillographs and resistance-capacity coupled amplifiers. Four channels have been available and the usual arrangement has been to record the EEG from the scalp with two of these channels and bipolar electrode placements over frontal, motor, and parietal regions of one side of the head; the other two channels being used to record from the flexor and extensor muscles in the thigh or upper arm of the opposite side. On other occasions the EEG has been recorded from the motor areas of the two sides and the muscle action potentials from the flexor or extensor muscles of the two sides in the upper arm or thigh. The patients have been examined whilst lying in the supine position on a blanket covered bed. This is important if the friction artefacts are to be avoided which occur during convulsive movements when a leather-covered couch is used.

OSCILLOGRAPHS.-The oscillographs used in this work are copies of those designed by Mr. A. M. Grass of Boston, who kindly gave permission for them to be made in this country as the shipping position at the time this work was started made importation impossible. The writers have a performance strictly comparable with that of the Grass type with the exception that 20 per cent. of the maximum linear amplitude of deflection hase been sacrificed to allow a small increase in the naturap period of the system; this lies between 70 and $80 \mathrm{c} / \mathrm{s}$ and is critically damped. The importance that is attached to the shape of waves that have components both above and below the resonant frequency of the recording system must be limited. The reason for this is that although the system may have a good response to the higher frequency components of a complex waveform, when these fall above the resonant frequency of the writer they may be shifted in phase in relation to any lower frequency components that fall below the resonant frequency. When such mutual phase shifts of the components of a complex wave occur the shape of the wave will be altered. Though the shape of a wave of the type mentioned may not be recorded accurately, differences in the shapes of waves will remain of significance with any one recording system. In spite of these relative inaccuracies at the higher frequencies it has been necessary to tolerate the inkwriting oscillographs rather than to use a more accurate recording system. Such a system with cathode ray oscillographs, giving four simultaneous records, has been available and has been used to monitor and check the performance of the writers in recording waves with periods of less than 10 to 12 milliseconds, which is about their extreme upper limit of accurate recording. Otherwise this second recording system has been of little value in these studies on account of the long intervals that may occur between the myoclonic outbursts which make photographic recording prohibitively costly. With the inkwriting oscillographs recording paper speeds of 4 and $8 \mathrm{~cm}$. per second have been used during the periods when jerkings were expected and $2 \mathrm{~cm}$. per second at other times. Time scales are indicated in seconds at the top of the records, and also in some cases, at the faster recording speeds, by a $50 \mathrm{c} / \mathrm{s}$ trace or a 0.1 second mark in the centre of the record. Calibration of amplification is indicated in microvolts $(\mu \mathrm{V}$.) or millivolts $(\mathrm{mV}$.) at the right-hand end of the records. The records throughout read from left to right.

AMPLIFIERS.-The amplifiers used are of conventional design and give the same amplification for all frequencies from 1 to $100 \mathrm{c} / \mathrm{s}$, with optional reduction of the 

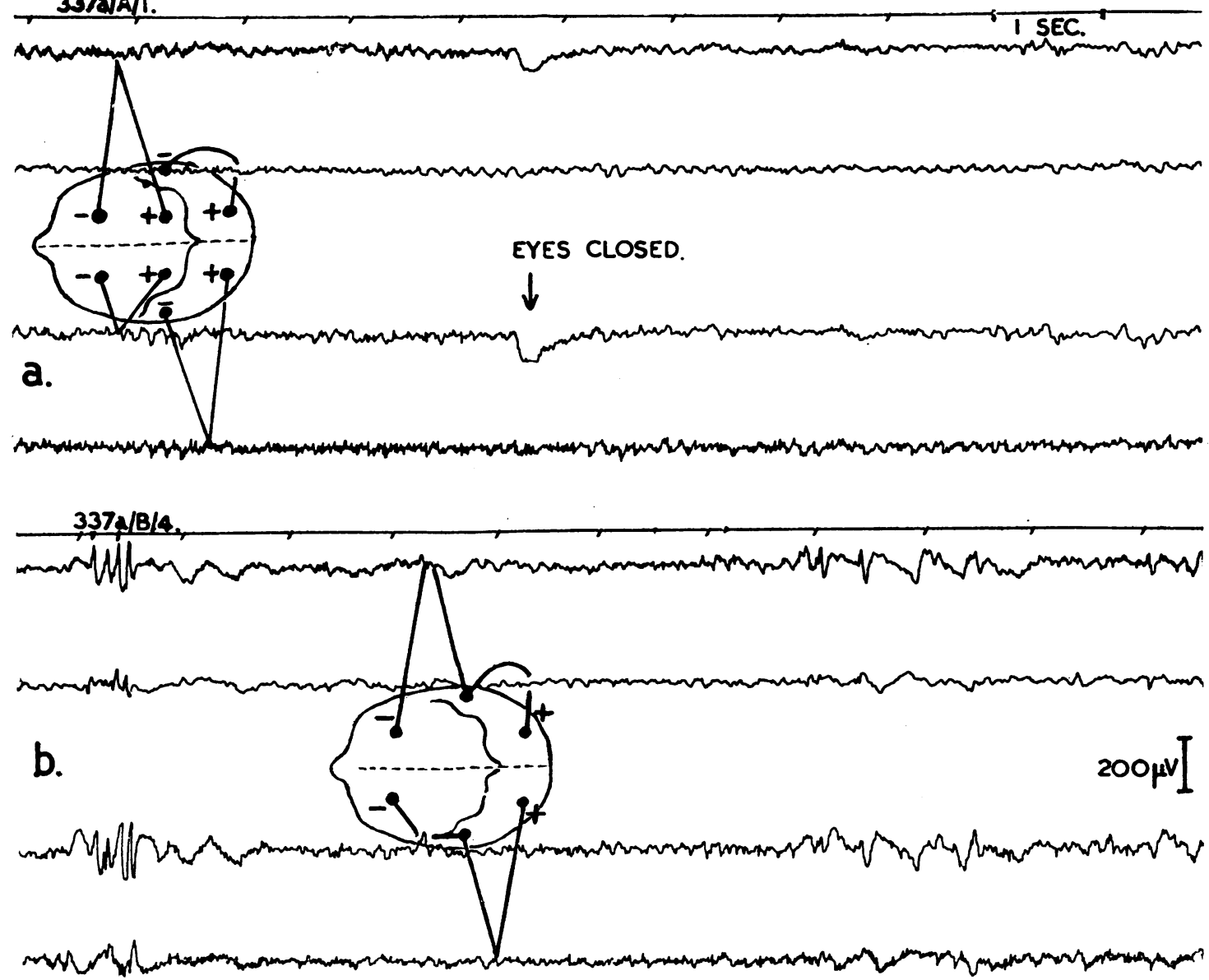

FIG. 1.-EEG records between seizures from a patient (337) with myoclonic epilepsy showing (a) rhythms at 6 to $7 \mathrm{c} / \mathrm{s}$ unaffected by closing the eyes, and $(b)$ groups of spikes and slow waves. In this and all following records the time scale at the top indicates one-second intervals. The + and - signs at the electrodes in all the head diagrams indicate that a potential difference such that the electrode marked + is positive with respect to that marked -, will cause an upward deflection in the record.

response to the higher or lower frequencies. The input connections are symmetrical with respect to earth and lead to an input circuit of the type described by Toennies (1938). In later experiments this circuit has been replaced by one designed on the principle described by Buchthal and Nielsen (1936) and Offner (1937), which has advantages when followed by pushpull amplifiers such as those used to drive the inkwriting oscillographs. With this circuit it is possible to record simultaneously from scalp and a limb without the electrocardiogram appearing in one or both records; it also prevents the muscle action potentials, which may be as large as 10 or $20 \mathrm{mV}$., from appearing in the EEG record, even when this is small and is being recorded with a high amplification.

The standard amplifier setting used for recording the EEG gives an equal response to all frequencies between $2 \mathrm{c} / \mathrm{s}$ and $50 \mathrm{c} / \mathrm{s}$. When the muscular jerkings are very severe low frequency movement artefacts may become a serious nuisance and under these conditions the response is kept constant from $50 \mathrm{c} / \mathrm{s}$ down to $20 \mathrm{c} / \mathrm{s}$ and then attenuated by 30 per cent. at $10 \mathrm{c} / \mathrm{s}$. The records taken with these last constants cannot be used for direct comparison of waveforms with those in records taken at the standard setting on account of the mutual phase shifts of the components of complex waves that occur; however, changes of the shapes of waves that occur in any one record remain significant. For recording the muscle action potentials amplifier settings are used which give an equal response to all frequencies between $20 \mathrm{c} / \mathrm{s}$ and $50 \mathrm{c} / \mathrm{s}$. Below $20 \mathrm{c} / \mathrm{s}$ the response is attenuated by 30 per cent. at $10 \mathrm{c} / \mathrm{s}$. Above $50 \mathrm{c} / \mathrm{s}$ the response is increased by 10 per cent. at $80 \mathrm{c} / \mathrm{s}$ and then falls steadily and is attenuated by 80 per cent. at $150 \mathrm{c} / \mathrm{s}$. The rise of the response at $80 \mathrm{c} / \mathrm{s}$ has been allowed on account of the considerable extension of the response to higher frequencies which it makes possible.

ELECTRODES.-The electrodes used to pick up the potential differences from the scalp have in all cases been similar to those described by Walter (1936). These consist of a gauze-covered sponge pad, $1 \mathrm{~cm}$. diameter, soaked in saline solution and supported on a chloridecoated silver tube. They have been held in place on the scalp by a rubber and whalebone cap. On account of the violence of the subject's movements during attacks smaller electrodes held in place by collodion might have been preferable. However, the time saved in application of the saline pad type of electrode has been of great importance and they have not in fact been found, when properly applied, to cause any significant artefacts.

For recording the muscle action potentials large surface plate electrodes, 2.5 by $3.5 \mathrm{~cm}$. in size, making contact through Cambridge electrode paste and held in place by rubber straps have been used. Two such 
plates separated by 1.5 to $3 \mathrm{~cm}$. have been applied to each muscle group to be studied. These large contacts have been used in preference to needle electrodes for two reasons. First, the needle electrode was introduced by Adrian and Bronk (1929) because of the very restricted space from which it picks up potential differences. Since these studies are concerned with the behaviour of muscle groups as a whole this restricted pick-up is undesirable. Secondly, the risk of damage to the muscle, or breakage of the needle during the jerks, is so high that to use such an electrode has been considered an unwarranted tax on the co-operativeness of the subjects. The chief disadvantage of the big surface plate electrodes used is the large area from which they pick up potential differences. In practice the localization of pick up given by these electrodes has been found to be satisfactory for this work. That is to say, when flexor muscles in the thigh or upper arm are contracting no potential differences of significant size appear in plate leads on the extensor aspect of the limb at the levels of amplification used in these studies. The same applies with regard to electrodes on the flexor muscles in the upper arm when the extensor muscles are contracting. In the thigh the muscle masses are less favourably disposed. When the quadriceps extensors are contracting action potentials may appear in plates over the hamstring muscles. These potentials are about 10 per cent. of the size of the action potentials picked up by plates, with a similar separation between them, applied over the contracting muscles themselves. Fortunately the order in which the potentials have been found to appear has made it possible to exclude this degree of spread from being important.

\section{Results}

\section{Myoclonic Epilepsy}

INTERSEIZURE RECORDS.-The two subjects who have severe myoclonic jerkings as a prodrome to their major convulsions show both similarities and differences in their resting EEG records. Both subjects show a bilateral discharge from frontal, parietal and occipital regions at 6 to $7 \mathrm{c} / \mathrm{s}$ which is unaffected by opening or closing the eyes. In one case (337) this discharge is rhythmical and rarely broken up by slower rhythms (Fig. 1 (a)). In the other case (407) the discharge is very irregular and is broken up by continuous slower discharges of small amplitude at frequencies down to $4 \mathrm{c} / \mathrm{s}$ (Fig. 2 (a)). Both subjects show outbursts of high voltage slow activity at 2 to $3 \mathrm{c} / \mathrm{s}$, but these are larger and more common in the second case (407).
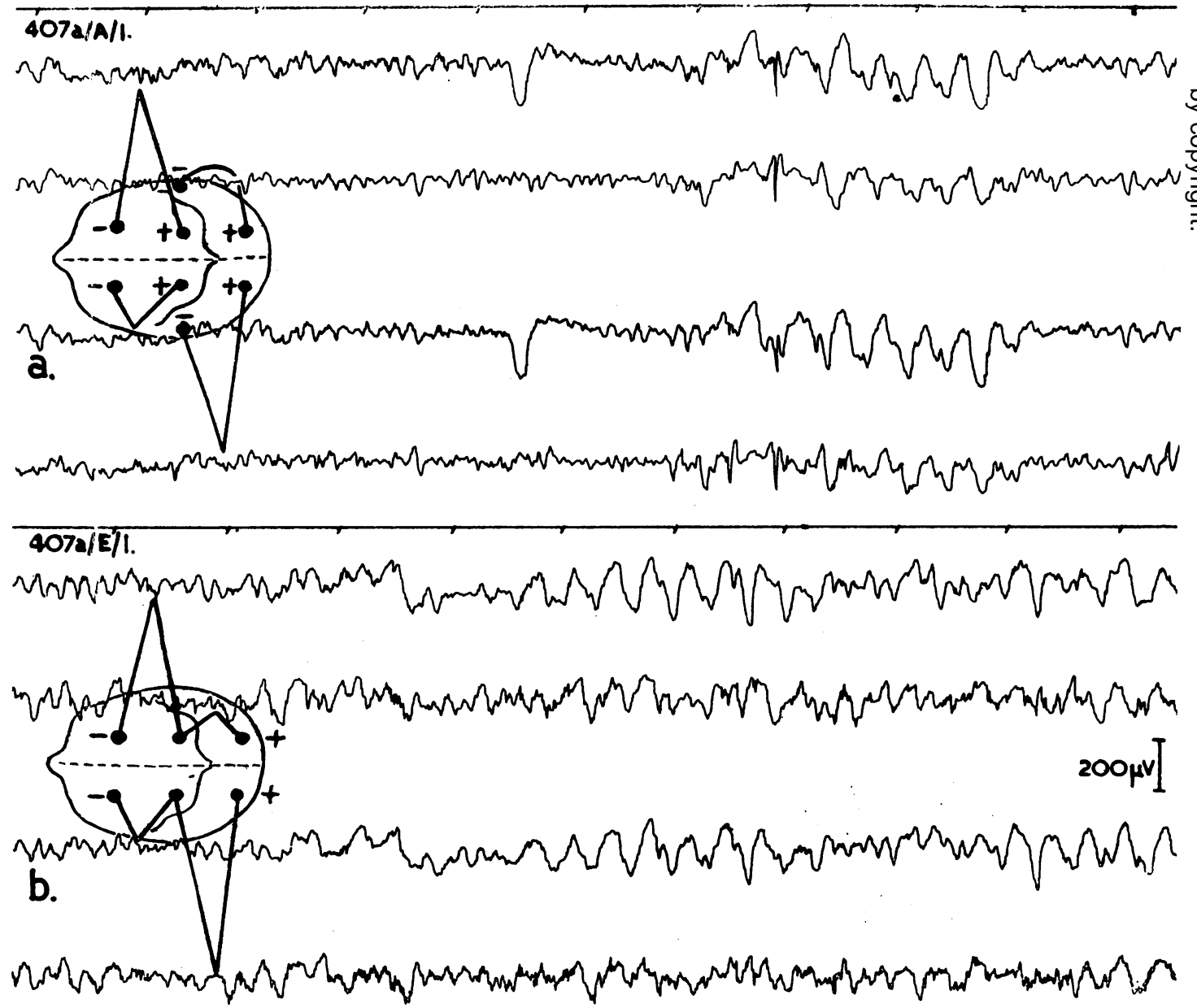

FIG. 2.- -EEG records between seizures from a patient (407) with myoclonic epilepsy showing (a) greater amount of slow activity at 2 to $4 \mathrm{c} / \mathrm{s}$ than in the records of Fig. 1, and $(b)$ effect of overbreathing in this case. 


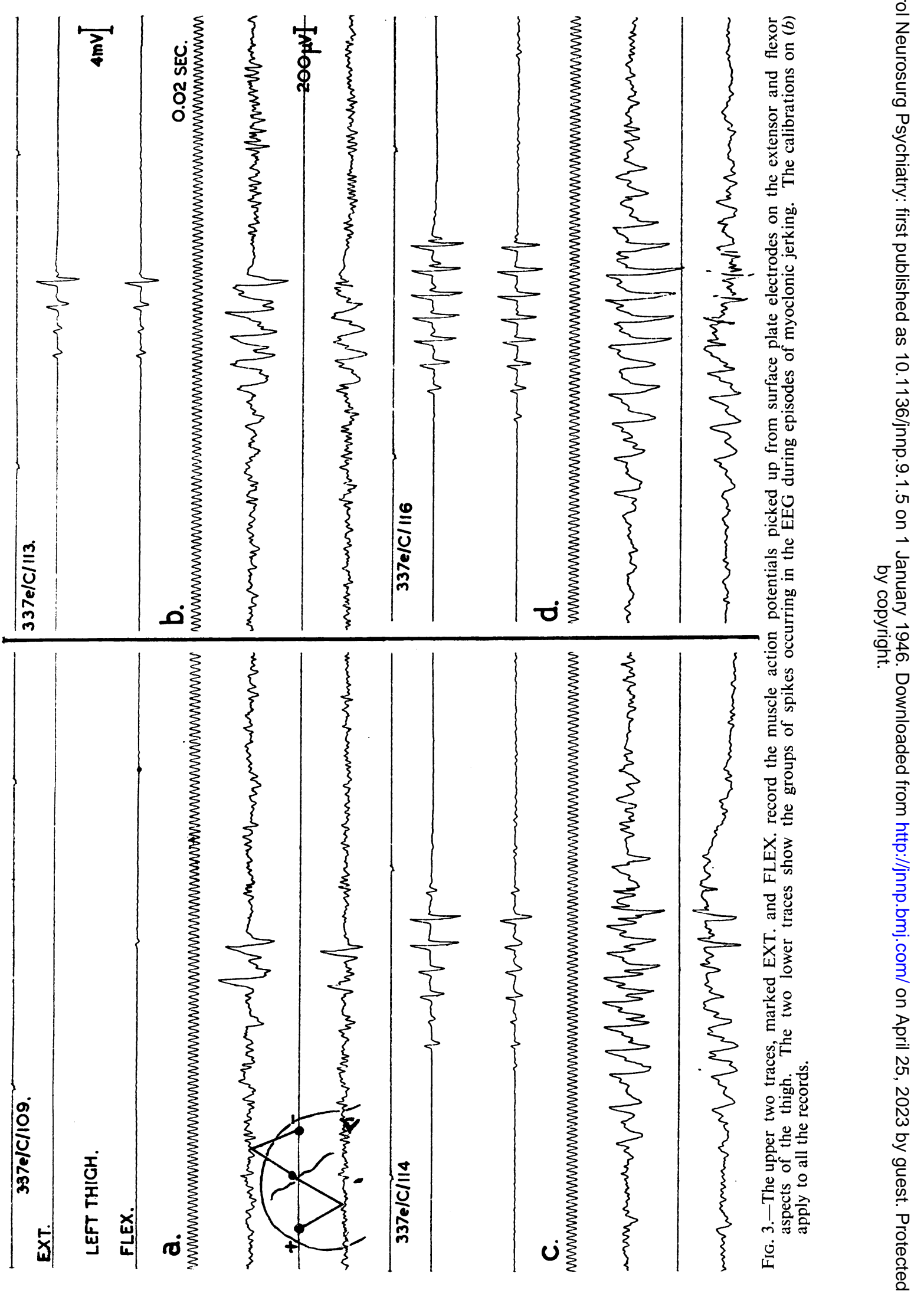


G. D. DAWSON

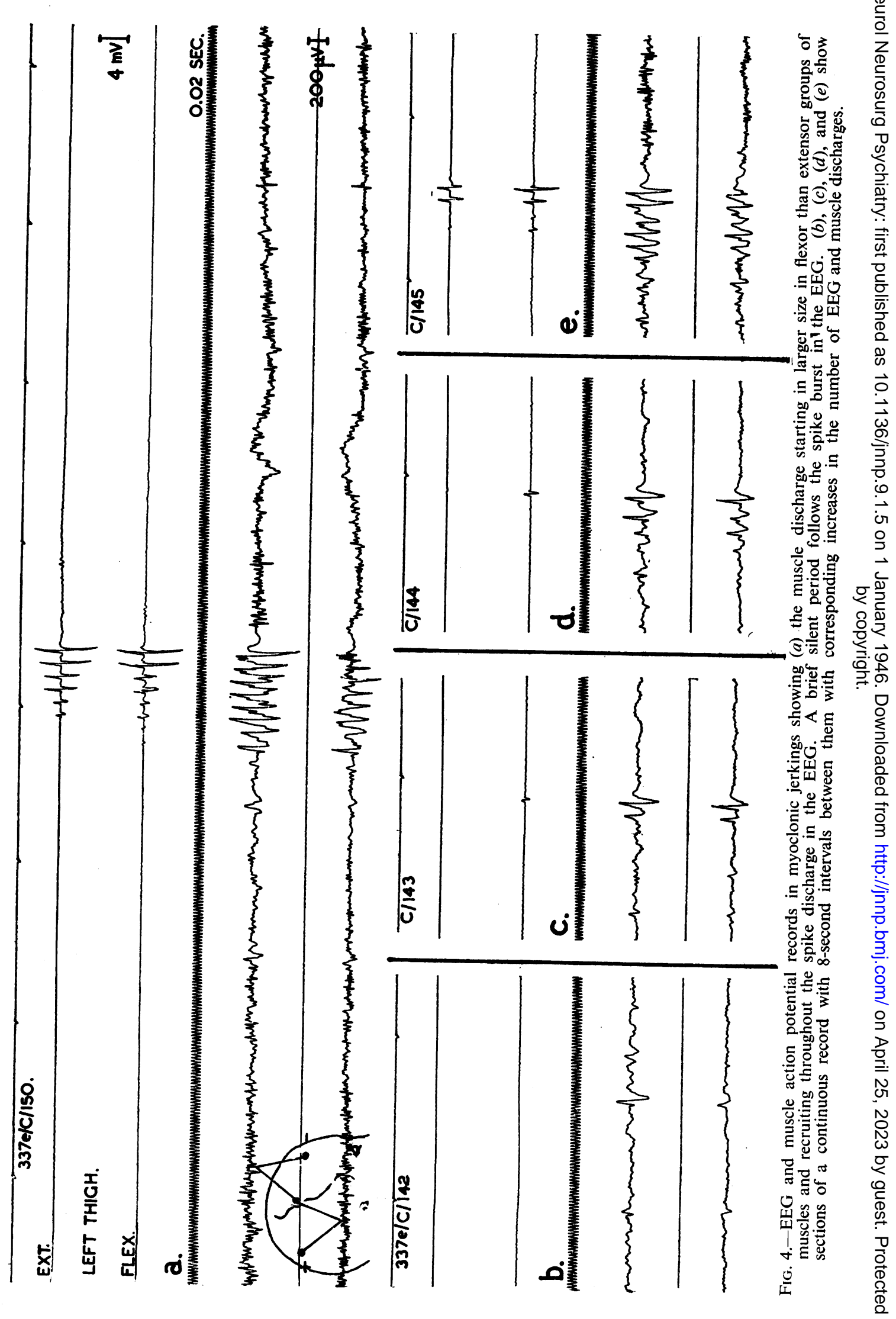


These slow waves are largest in the frontal regions and may be accompanied by discharges of single or multiple spikes (Figs. $1(b)$ and $2(a)$ ). In the quiescent periods muscular jerkings do not accompany these spikes. In the first case during overbreathing no effect on the slow waves is seen, but in the second case the slow waves are greatly increased in number (Fig. $2(b)$ ). In neither case has overbreathing been found to affect the frequency with which the groups of the spikes occur. The discharges present in these two cases are usually symmetrical on the two sides of the head.

RECORDS DURING SEIZURES.-D During the episodes of jerking the EEG takes on an unusual and highly characteristic appearance. In this state brief groups of spikelike discharges occur at a frequency of 8 to 13 per second. The term spike will be used to describe these waves since they appear sharp at slow speeds of recording, though at the higher speeds used for measuring time relations they often lose much of their spikelike character. The spikes in the EEG may occur singly or in groups (Fig. 3 $(a)$ to $(d)$ ). Although the spikes are usually larger in frontal than in precentral leads no case of jerking occurring synchronously with the spikes in the EEG has been seen when they have not appeared in the precentral leads. The duration of the EEG spikes is variable from about 25 to $75 \mathrm{msec}$. and is related to the placing of the electrodes with respect to the potential gradients on the scalp, and to the place of the spike in its group (Fig. 3). Over the duration of one burst the shape of the spikes changes in a way that tends to repeat itself in any one subject. The first phase of the potential change is surface negative and this is followed by a second phase that is surface positive. Sometimes a small surface positive change precedes the surface negative one (Fig. $4(b)$ and $(d)$ ), but this is not constant. Towards the end of the burst the discharge appears as a short duration surface positive wave alone, or a rectangular surface negative wave. This change of shape may be due either to a movement of the origin of the potential difference with respect to the electrodes or to an actual change in the form of the potential difference. Which of these alternatives is the correct one cannot be decided in the absence of fuller studies of the potential distributions in the attacks. Following the spike burst there is usually a "silent period" of 0.1 to $0.5 \mathrm{sec}$. during which electrical activity falls to a minimum before returning to the same level that it had before the spike burst (Figs. $3(a)$ and $(b)$, and $4(a)$ ).

Associated with these discharges there occur the massive muscular jerkings found in this type of myoclonic epilepsy. With the amplifications used no muscle action potential or detectable muscular jerk has been found to accompany a single isolated spike in the EEG. Two spikes are usually accompanied by a minimal action potential in flexor muscles alone, whilst when a longer burst of spikes occurs action potentials most commonly appear first in the flexor muscles, follow in the extensor muscles and recruit steadily up to the end of the spike burst, then cease suddenly. A series of records in Fig. $4(b),(c),(d)$, and $(e)$, shows a progressive increase in the number of spikes in four consecutive bursts, with a corresponding increase in the number and size of the muscle action potentials. The length of the "silent period" or period of " extinction" after the spike burst also increases with the number of spikes in these records. The sections shown in Fig. $4(b),(c),(d)$, and $(e)$, are parts of a continuous record with intervals of 8 seconds between them in which no other equally large paroxysmal disturbances occur. They suggest a rapid and progressive increase in the cerebral abnormality over a period of 30 seconds. Once both the flexor and extensor muscles are contracting their action potentials are, within the accuracy of the measurements possible with these methods, apparently synchronous and without any sign of reciprocal innervation.

It was stated above that when a single isolated spike occurs in the EEG it has not been seen to be accompanied by a muscle action potential or clinically obvious muscular contraction. However, if the single spike follows within about 1.5 or 2 seconds of a previous burst of spikes, apparently some of the facilitation that has been built up during the burst remains, and a jerk and action potential, such as that indicated by the arrow in Fig. 5, may occur in the flexor muscles alone. For an extensor discharge to occur with a single spike it seems to be necessary that the interval between that spike and a preceding burst shall not be more than 0.5 seconds. If a break in the spike discharge in the EEG occurs, when the discharge restarts there has been a greater decay of facilitation for extensor than for flexor mechanisms. The records in Fig. 6 show that after an interval of 0.3 seconds the facilitation for the flexors has fallen little, and the muscle discharge restarts at the size at which it ceased (Fig. $6(a)$ ), or reduced by 50 per cent. (Fig. $6(b))$. For the extensors, however, the facilitation has apparently decayed to a greater extent, as the first of the succeeding discharges is much smaller than the last of the preceding onis. Some facilitation for the extensors remains, since the action potentials recruit more rapidly than in the initial burst. Measurements of the delay between the beginning of the EEG disturbance and the muscle action potential suggest a value for this not less than 15 and not more than $40 \mathrm{msec}$. These measurements, however, have to be accepted with caution for several reasons. First it is only in records such as those of Figs. $3(a)$ or $4(c)$ or $(d)$, that it is possible to decide where the EEG disturbances begin, and secondly it is difficult to be sure that the particular EEG spike used as a reference is associated with a particular muscle action potential. Higher recording rates help little, as it is found that when the waves are opened out by this means they have no salient features from which more accurate measurements may be made.

One point of interest in the records of the muscle action potentials during the myoclonic jerkings" is 


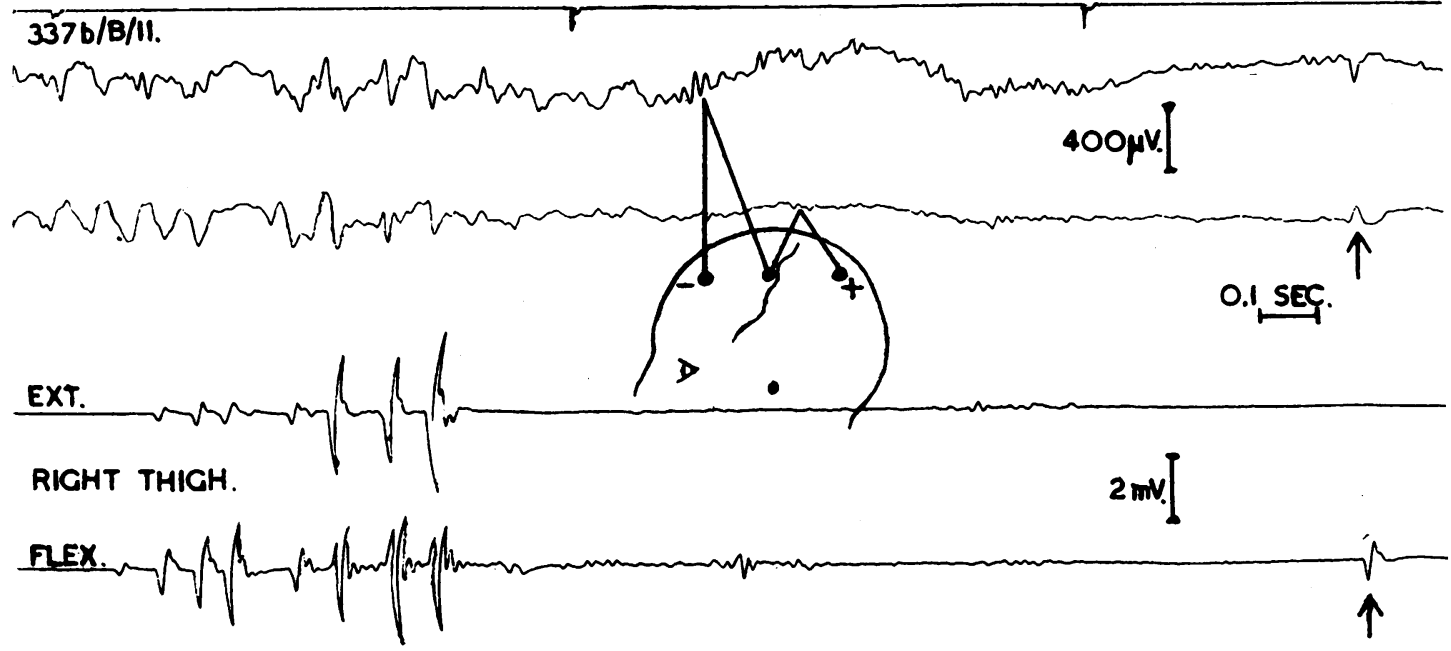

FIG. 5.-A single spike discharge in the EEG, marked by the arrow in the record, is accompanied by a muscle discharge if it follows within 2 seconds of a previous spike burst, cf. Fig. 4 (b) where a single spike not following a previous burst is unaccompanied by a muscle discharge.
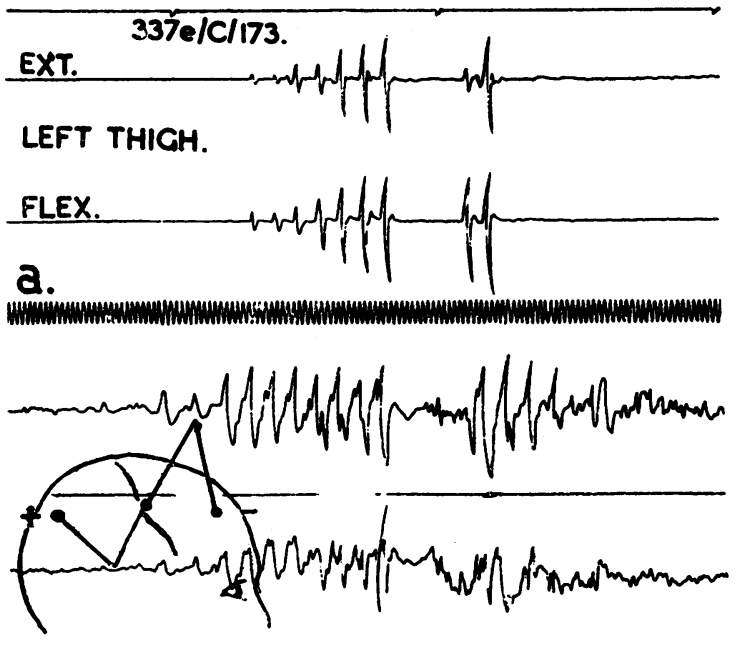

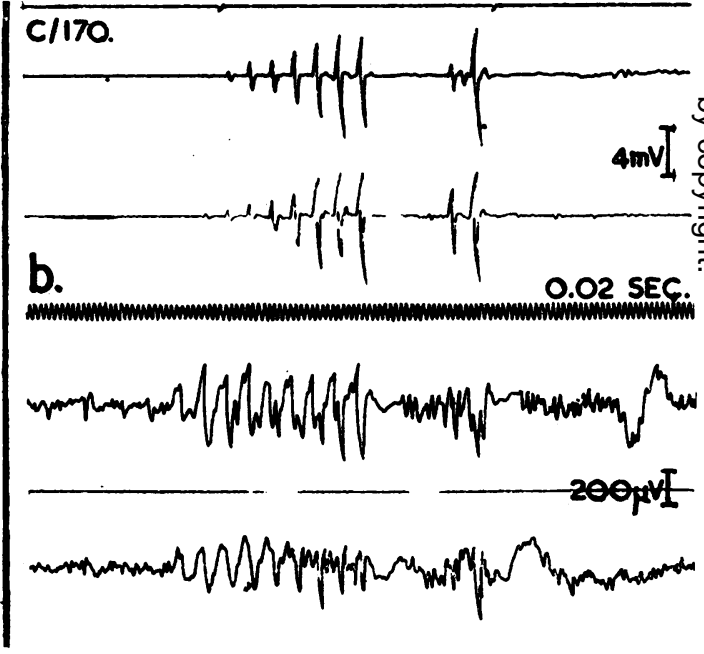

FIG. 6.-Following a break in the EEG discharge the muscle action potentials restart with the size of those in the flexor groups reduced less than that of those in extensor groups.

their extreme simplicity and brevity, and the apparent absence of after-discharge. The method of recording used might possibly introduce as an artefact an apparent lengthening of the potential differences; it is highly unlikely to shorten their apparent duration. The recorded action potentials have neither more phases nor greater duration than those evoked by eliciting a reflex jerk with a tendon tap. It does not seem to be possible to produce anything as brief as this by voluntary movements of even the shortest duration. This seems to indicate some highly synchronized form of centrifugal discharge in the myoclonic state. After this stage of the seizure, characterized by the intermittent episodes of jerking, has been in progress for about one or one-and-a-half hours one of the jerks passes, with a pause of usually not more than 2 or 3 seconds, into the tonic stage of a major tonic-clonic convulsion (Fig. 7). It is notable that although the tonic stage of the seizure is well developed by the end of the second part of the record, which is continuous with the first part, there is only a low voltage fast discharge in the EEG and the rate of this, 45 to $50 \mathrm{c} / \mathrm{s}$, suggests that it is of muscular rather than cerebral origin. The absence of the 20 to $25 \mathrm{c} / \mathrm{s}$ discharges usually associated with the start of a Grand Mal seizure (Gibbs, Gibbs and Lennox, 1937) has been noted on several occasions in these subjects.

EFFECTS OF SENSORY STIMULATION.-In the period of prodromal muscular jerkings before the major seizure two stages may be distinguished clinically. 

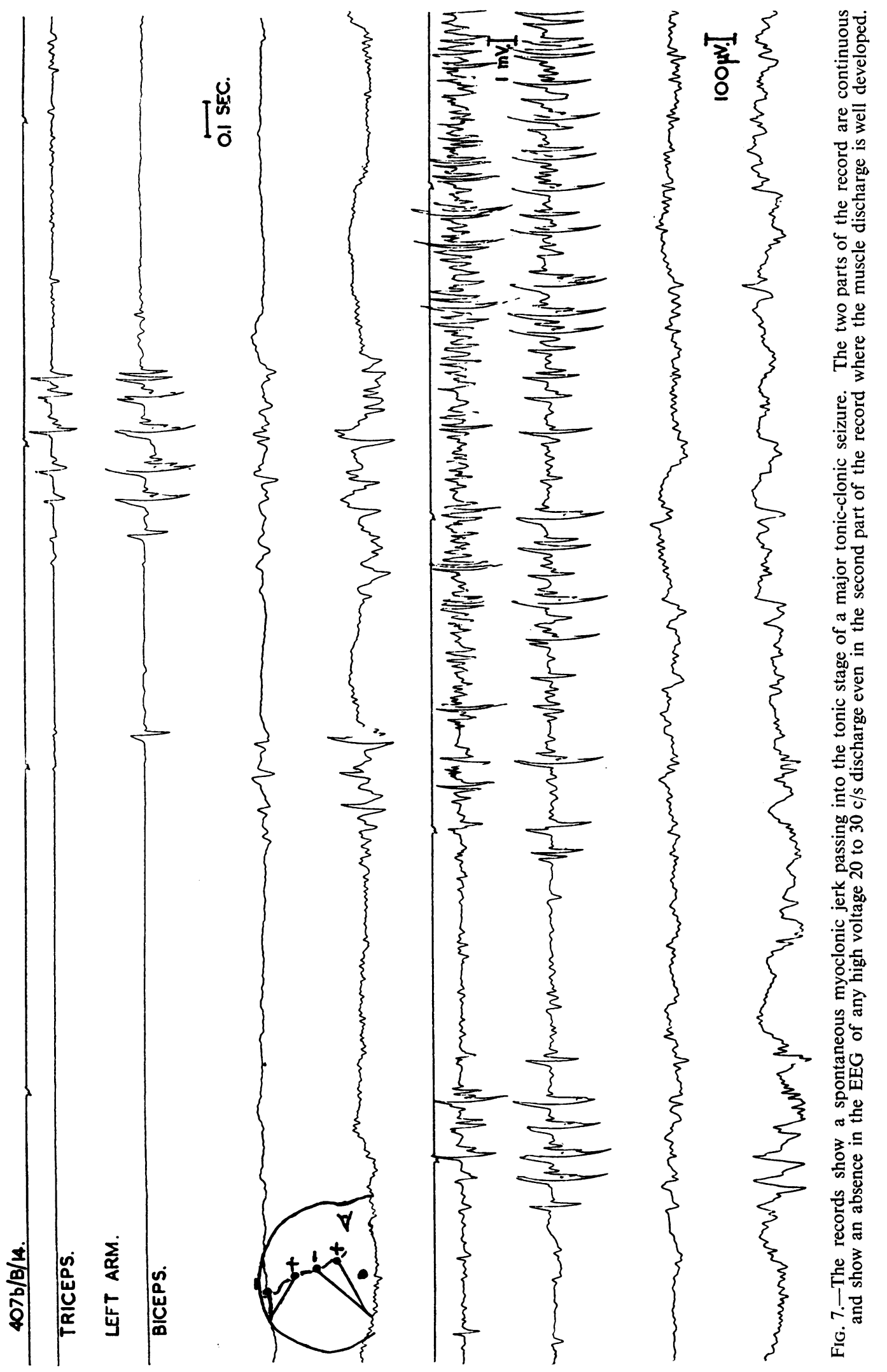

c

2 
In the first stage, if a knee jerk is elicited the initial, normal response is followed by another jerk. The interval between the two jerks varies between 80 and $180 \mathrm{msec}$. and the second jerk is usually confined to the muscles stimulated, but may occasionally occur in the antagonists (Fig. $8(a)$ to $(e)$ ). In this stage no correlate of the jerk is found in the EEG. The action potentials show that the initial jerk, marked $\mathrm{KJ}$ in the records, is simple and brief like that in a normal tendon reflex contraction. The action potential of the secondary jerk, marked by the arrow on the records, has a different character: it is multiphasic, increases in size slowly up to a maximum, then declines slowly and shows a considerable dispersion in time. The record showing the shortest latency for the secondary action potential (Fig. $8(d)$ ) was made on a different day and with different amplification from that showing the longest latency (Fig. $8(a)$ ). The difference in the amplifications used does not account for the difference in the latencies as, although some of the earlier potentials in the secondary discharge in Fig. 8 (a) may not be visible on account of the lower amplification used for that record, the discharge reaches its maximum $80 \mathrm{msec}$. later there than in 0 the record of Fig. $8(d)$. It does not seem to be 0 possible to correlate the latency or the size of the secondary discharge with the size of the initial jerk action potential. The smaller of the two figures for the latency of the secondary jerk $(80 \mathrm{msec}$.) is significantly less than a normal reaction time; of therefore, unless the reaction time is considerably shortened in this condition, it is unlikely that the secondary response is a voluntary one to a stimulus that has assumed a startling nature. The secondary response has many of the characters, in a greatly exaggerated degree, of the rebound that occurs normally at the end of the inhibitory silent period

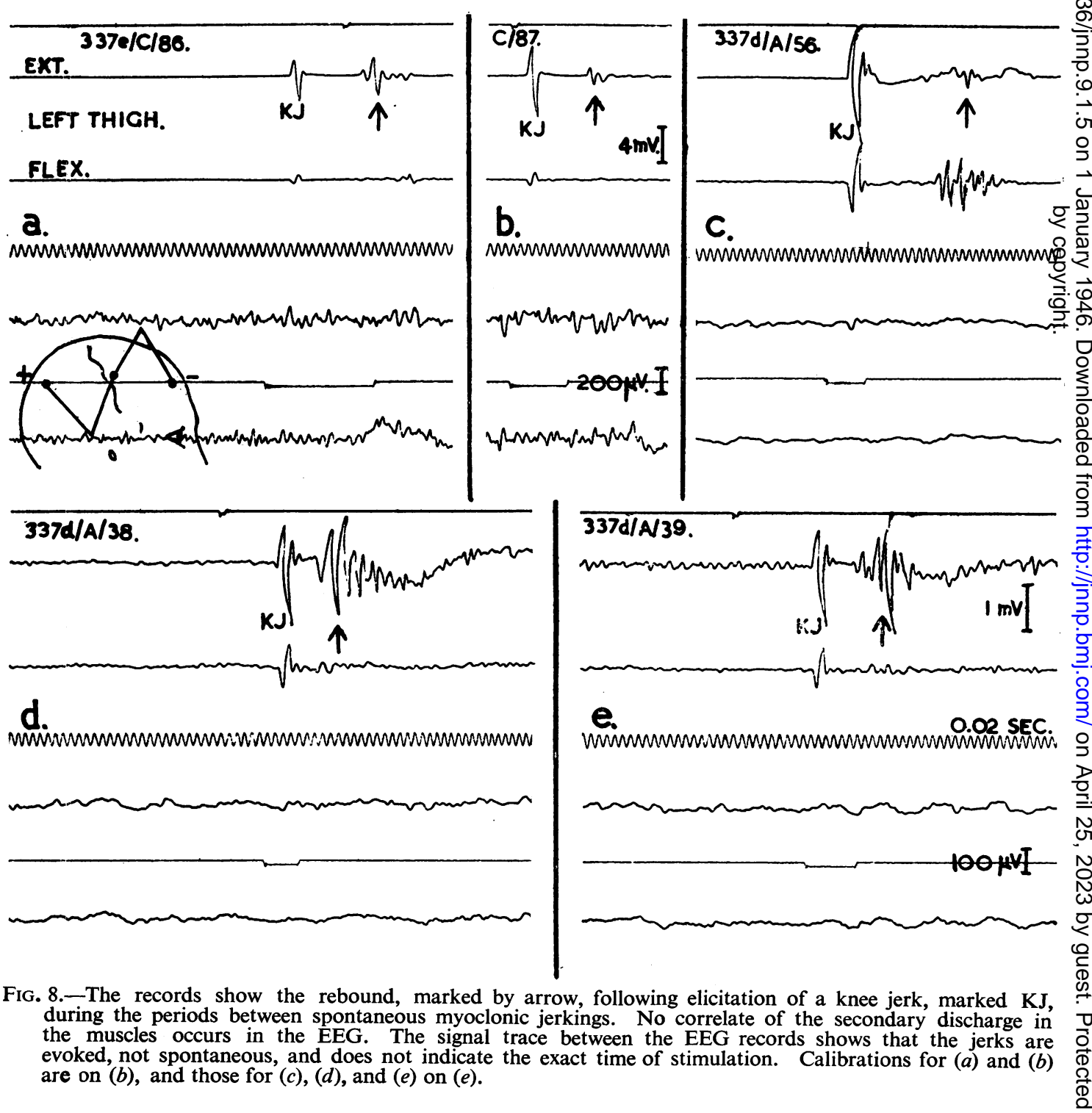


EXT.

\section{LEFT THIGH.}

sopVI

FLEX.

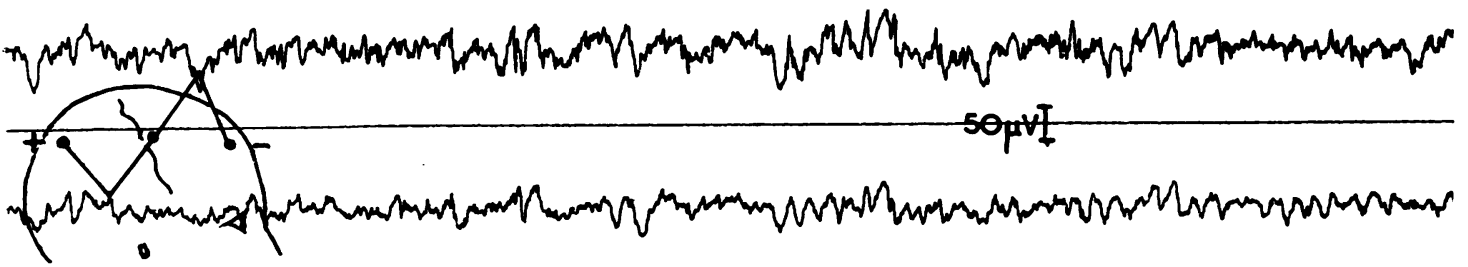

FIG. 9.-Records from the muscles of the leg at higher amplifications show an abnormally large amount of resting fasciculation between the episodes of myoclonic jerking. No correlate of the fasciculation appears in the EEG.

following a tendon reflex. In the normal person the rebound jerk has an action potential that does not exceed one-tenth of that of the primary jerk, whereas in this stage of the myoclonic state it is often as big as, and sometimes greater than, that of the primary jerk. Also in the normal person a large primary jerk is usually associated with a longer period of inhibition than that which follows a small primary jerk.

In the records shown in Fig. 8 a comparatively large potential difference is picked up in the flexor surface electrodes at the time when the extensor action potential of the initial reflex jerk occurs. Some of this potential difference may be due to the direct electrical leakage mentioned in the section on methods. That the major part of the potential difference is not due to leakage is suggested by the absence of any large flexor surface potential during the presence of the secondary extensor jerk potential, even though this is larger than the primary discharge on two of the occasions shown (Fig. 8 $(d)$ and $(e)$ ). If this flexor surface potential difference is arising in flexor muscles, and it seems that this possibility needs to be considered, it must either be excited by the same afferent volley that leads to the extensor discharge, or by the afferent volley excited by the stretching of the flexor muscles at the time the extensor muscles contract. Measurements show a small but relatively constant delay of 5 to $10 \mathrm{msec}$. between the beginning of the extensor action potential and that of the flexor muscle. This apparent delay is probably not due to any lack of alignment in the oscillographs, errors due to this cause in these records being less than $5 \mathrm{msec}$. at the recording speed used. This order of delay suggests that the second possibility is the less likely of the two. Without more accurate timing methods it is unwise to draw any conclusions from this finding but, since it is clear that when the myoclonic jerkings occur there is apparently a failure of reciprocal innervation (Figs. 3 and 4), it may be suggested that a similar failure can also occur to some extent in this earlier stage when spinal reflex excitability is increased. That the failure of reciprocal innervation in the myoclonic jerk is not just an artefact produced by electrical leakage of extensor surface potential differences to the flexor surface electrodes is clear, because the flexor surface potential differences commonly appear before those from the extensor aspect of the limb, and also they may increase in size when the extensor potentials are not altering (Fig. 5).

If recordings are made with a high amplification from either flexor or extensor muscles at this stage of the attack an abnormally large amount of resting fasciculation is found (Fig. 9). This fasciculation is irregular and shows no tendency to become organized into bigger jerkings. No correlate of the fasciculation is seen in the EEG. This may possibly be another manifestation of the increased motor neurone excitation shown by the increased reflex excitability and rebound mentioned above.

In the second stage of the prodromal state that may be distinguished clinically, the reflex response to a tendon tap is followed by a generalized myoclonic jerk of the same type as those occurring spontaneously. These jerks may also be provoked by sudden unexpected noises, but a tendon tap seems to be a particularly adequate stimulus. During this stage a clear correlate of the jerk is found in the EEG (Fig. $10(a)$ to (c), which shows the effect of eliciting a triceps jerk in the left arm. The spike potentials that occur in the EEG are the same in general characters as those occurring spontaneously. The delay between the start of the tendon reflex action potential, marked $T J$ in the record, and the first significant wave in the EEG varies, in the records shown, between 130 and $150 \mathrm{msec}$; a similar range of values has been found in other experiments. In the record of Fig. $10(c)$ 
the first jerk elicited was unaccompanied by any cerebral spikes. A spontaneous spike on both sides follows, suggesting a spontaneous rise in excitation, and the next tendon tap is followed by a series of spikes and muscular jerks after an interval of $140 \mathrm{msec}$. The records of Fig. 10 seem to show that the cerebral discharge starts in larger size on the side of the head contralateral to the limb stimulated than that ipsilateral to it. This result has not been found with sufficient constancy to be accounted significant, but two records taken on the same occasion under the same conditions, when a stimulus has been applied by coincidence after a spontaneous discharge has already begun (Fig. $11(a)$ and (b)), show the cerebral discharge starting in a more nearly symmetrical fashion. This patient on some occasions had a tendency to asymmetry of the discharges and before this may be taken as evidence that the tendon tap evokes a discharge in the contralateral cerebral cortex first, further controls are needed. Both clinically and from the point of view of the EEG this stage is suggestive of the condition of an animal to whose cortex a subconvulsive quantity of a convulsant drug has been applied.

\section{Myoclonus with Wave and Spike Discharge}

INTERSEIZURE RECORDS.-The subject who has attacks of mild muszular jerkings that last for 24 to $D$ 48 hours shows in his EEG between these episodes a main rhythm that varies between 10 and $16 \mathrm{c} / \mathrm{s}$, that is symmetrical on the two sides, largest in occipital leads, and that disappears on opening the eyes. In addition there is low voltage activity at $4 \mathrm{c} / \mathrm{s}$ from all parts of the head. Overbreathing pro- 0 duces a paroxysmal discharge at 6 to $8 \mathrm{c} / \mathrm{s}$ all over 듬 the head, followed by an outburst of waves and $\frac{\bar{m}}{\sim}$ spikes of regular form lasting up to 12 seconds. This outburst is accompanied by mild clonic jerkings of all the limbs and a failure to respond to orders.

RECORDS DURING SEIZURES.-At the start of the prolonged periods of jerking the EEG shows a continuous discharge of slow waves and irregular

\section{7d/A 74.}

\section{R. TRICEPS Br.}

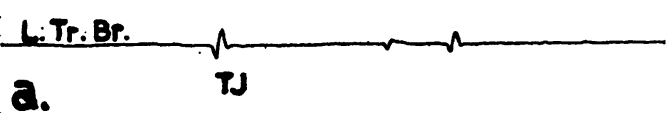

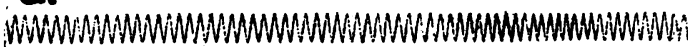
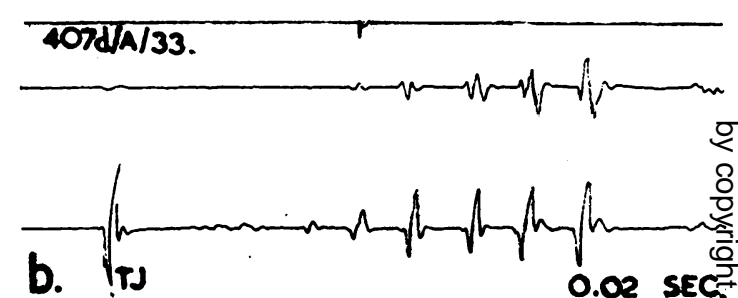
0.02 SEC?

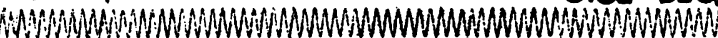

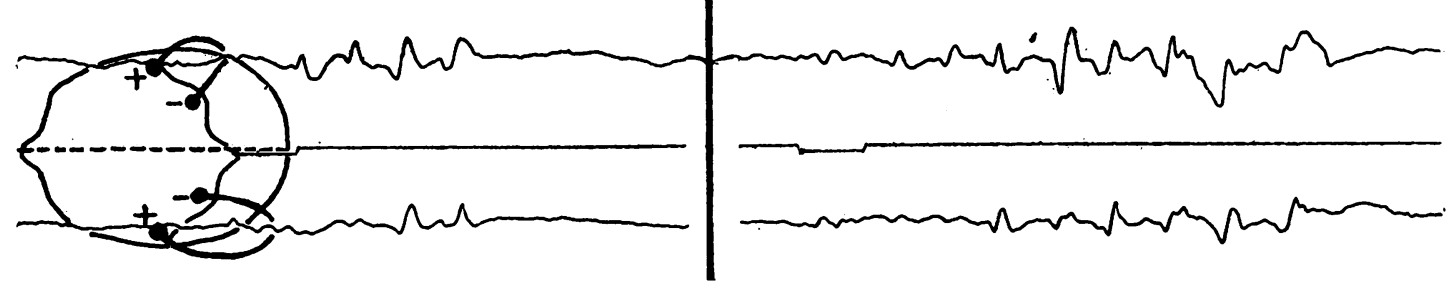

$4074 / 1 / 178$

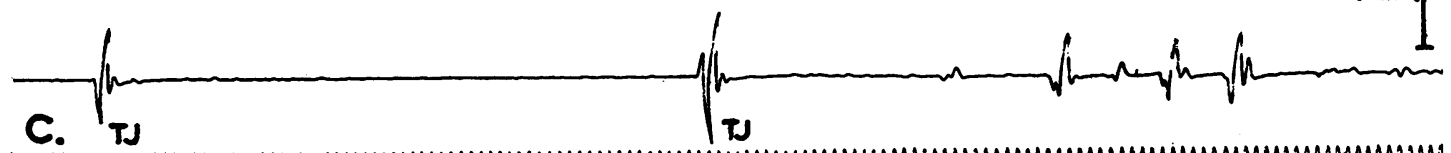

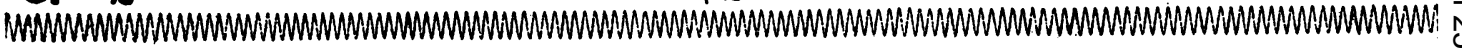

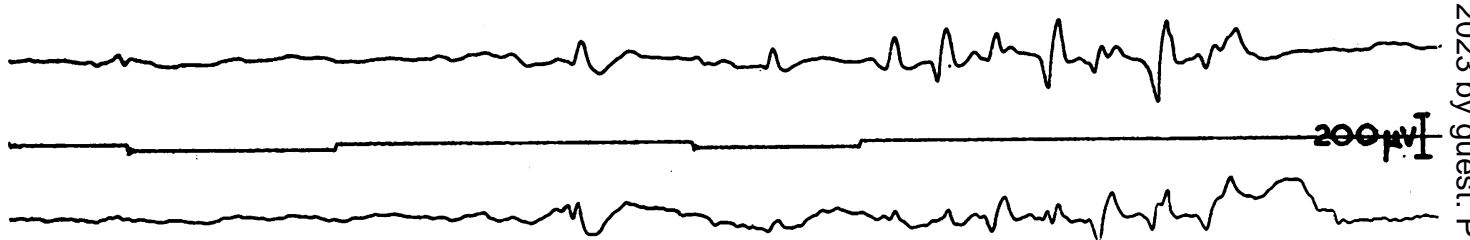

FIG. 10.-Records from the triceps and biceps muscles of the arm to show myoclonic jerkings with spikes in the EEG following the elicitation of a triceps jerk, marked TJ. This occurs at a later stage of the myoclonic state than the effect shown in Fig. 8. 
R. TRICEPS Br.

ind $P$ H

‥ I. B.

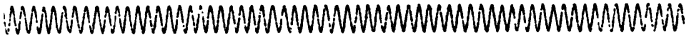

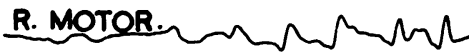

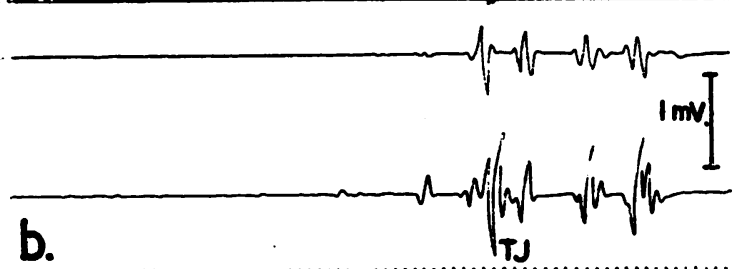

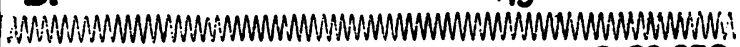
O.CQ SEC.

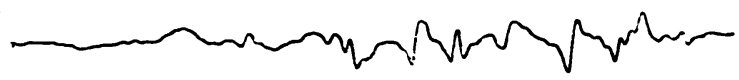

200pif.

L.MOTOR.

nothong

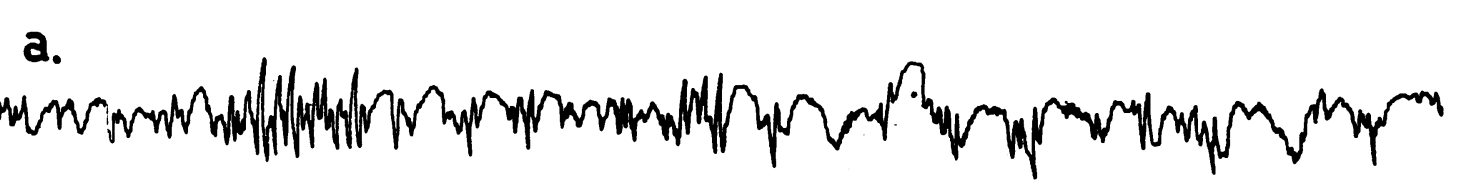

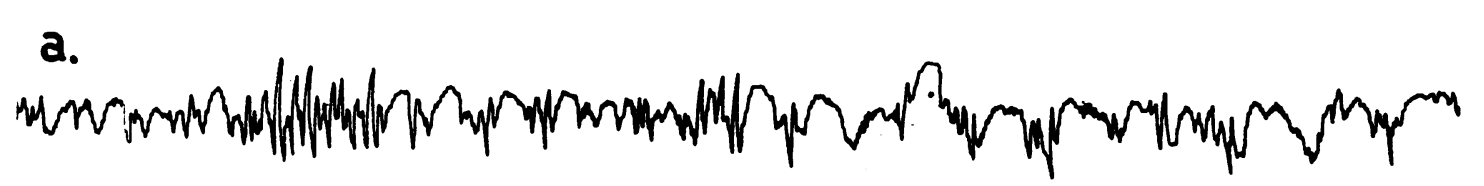

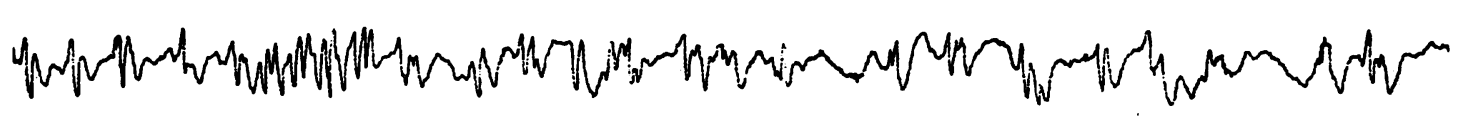
taneous jerk has already begun. The EEG record is more symmetrical than in the records of Fig. 10. where the EEG discharge follows the triceps jerk.

377ादि/1.

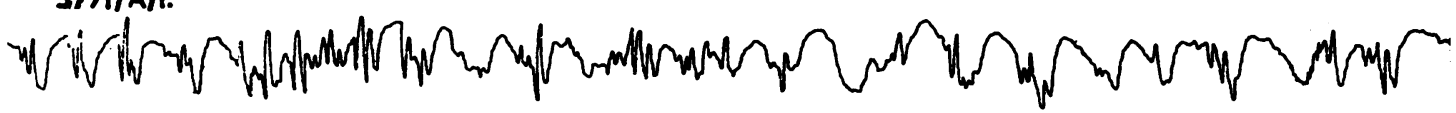

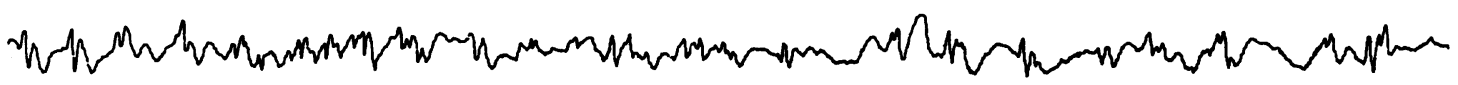

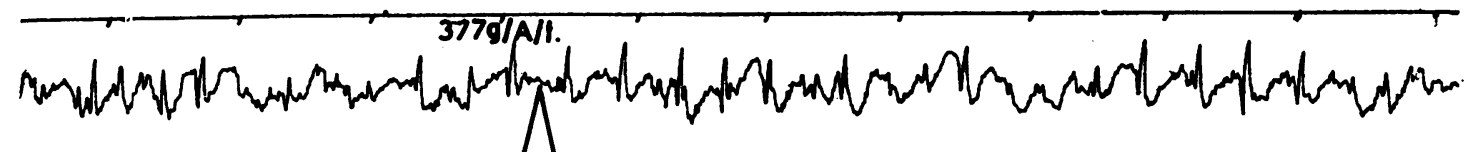
b.

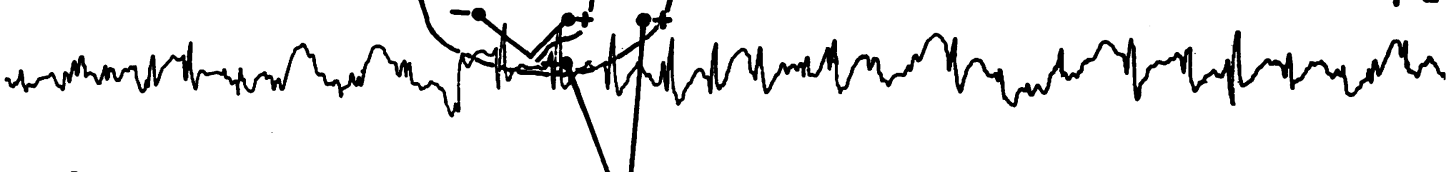

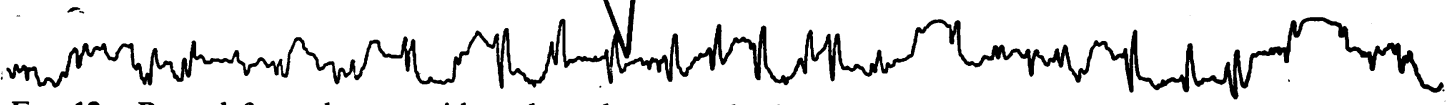

FIG. 12.-Record from the case with prolonged wave and spike discharge with myoclonus. (a) At the beginning of the attack. (b) Twenty-four hours later when the discharge has become more regular. Calibrations and
electrode placements are the same for the two records. 


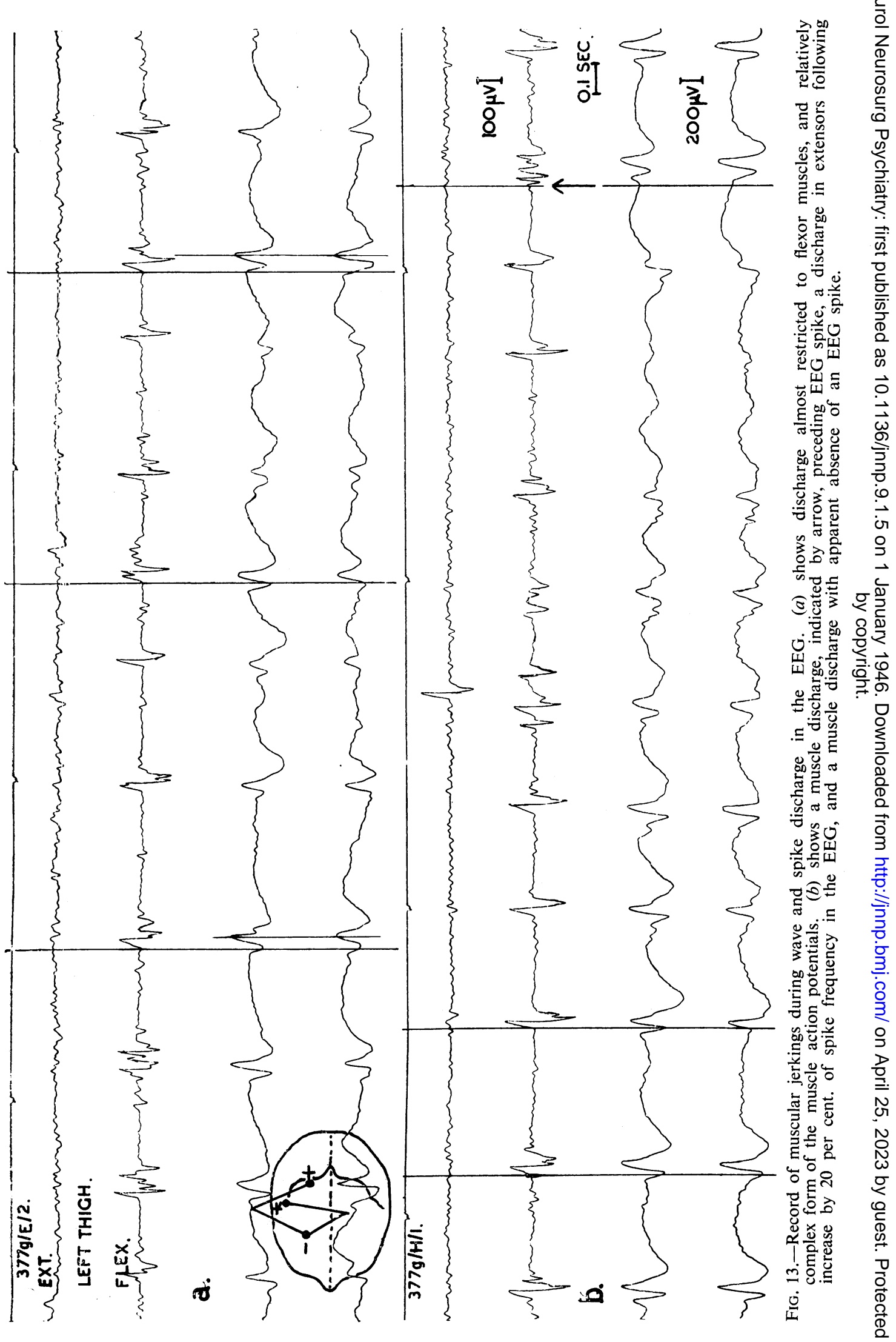


spikes (Fig. $12(a)$ ) over all parts of the head. This stage is characterized by diffuse and irregular muscular twitching in all parts of the body and some clouding of consciousness. After about five or six hours the EEG shows a more regular discharge of wave and spike form (Fig. $12(b)$ ), and the jerking becomes regular and synchronous in different parts of the body. In this stage there is no great reduction in consciousness and, though the subject may be euphoric and frequently whistles tunes, he is able to answer questions intelligently. When this type of discharge has become established it may persist, with little change except some increase in regularity, for 24 hours at 2.5 to $3 \mathrm{c} / \mathrm{s}$, symmetrical on the two sides and with amplitude up to $800 \mu \mathrm{V}$.

The muscular jerkings in this condition are clinically very slight compared with those occurring as prodromata of a major seizure, described in the last section. Records of muscle action potentials also show considerable differences between the two types. The discharges in this condition are still brief and may occasionally be as short as those in the myoclonic jerkings, but usually they are longer, have more phases, and an altogether less simple form (Fig. $13(a)$ and (b)). In addition the muscle discharge may remain localized to the flexor muscles, where it first appears, with only occasional discharges in the extensors. The time relations of the EEG spike and the muscle action potential appear to be more variable in this condition than in the more severe myoclonic state. In that state the muscle action potentials have never been found to precede the cortical wave and usually are delayed after it by not less than $15 \mathrm{msec}$. In the wave and spike seizure the muscle action potential does not seem to be so constantly delayed and may actually precede the earliest recognizable phase of the EEG spike (Fig. $13(b)$ at the point marked by the arrow.) This suggests that in the wave and spike seizure the efferent discharge giving rise to the muscular activity is not a direct correlate of the EEG spike, but that both are manifestations of some process that reaches its peak at the time they occur. That the presence of a detectable cortical spike is not necessary for the occurrence of a muscle discharge in the wave and spike seizure is also suggested by the later part of the record in Fig. $13(b)$. In this case it cannot be ruled out, however, that the apparent absence of the cortical spike may be due to the electrode arrangement used, and a movement of the area of greatest cortical activity with respect to the electrodes; this is suggested by the slow decline and return of the EEG spike at that part of the record.

The difference in size of these muscle action potentials when compared with those in the myoclonic prodrome is striking. In the latter state, with the electrode arrangements used, the potential differences may be as much as $10 \mathrm{mV}$. Under the same recording conditions the potentials during the wave and spike seizure rarely exceed $200 \mu \mathrm{V}$. The size of the spikes in the EEG is similar to that in the myoclonic prodrome, their duration is of the same order, and the same areas of cortex are discharging abnormally. Some other factor than these must therefore be sought to account for the small size of the muscle discharges in this wave and spike seizure, and their absence or even smaller size, in most seizures of this type. It may be that one factor is the longer interval between the efferent discharges in the wave and spike seizure. Since these discharges seem to reach their peak at about the time the spike occurs in the EEG they do not approach within less than about 0.3 of a second of one another. In the myoclonic prodrome it was seen that during such a period some decay will occur of the facilitation associated with a preceding spike or spikes. Spikes appearing at a rate of three per second, and not preceded by a burst at a faster rate, may not be associated with the building up of sufficient excitatory state to cause a larger discharge in the muscles. That this factor may possibly be an important one is suggested by the
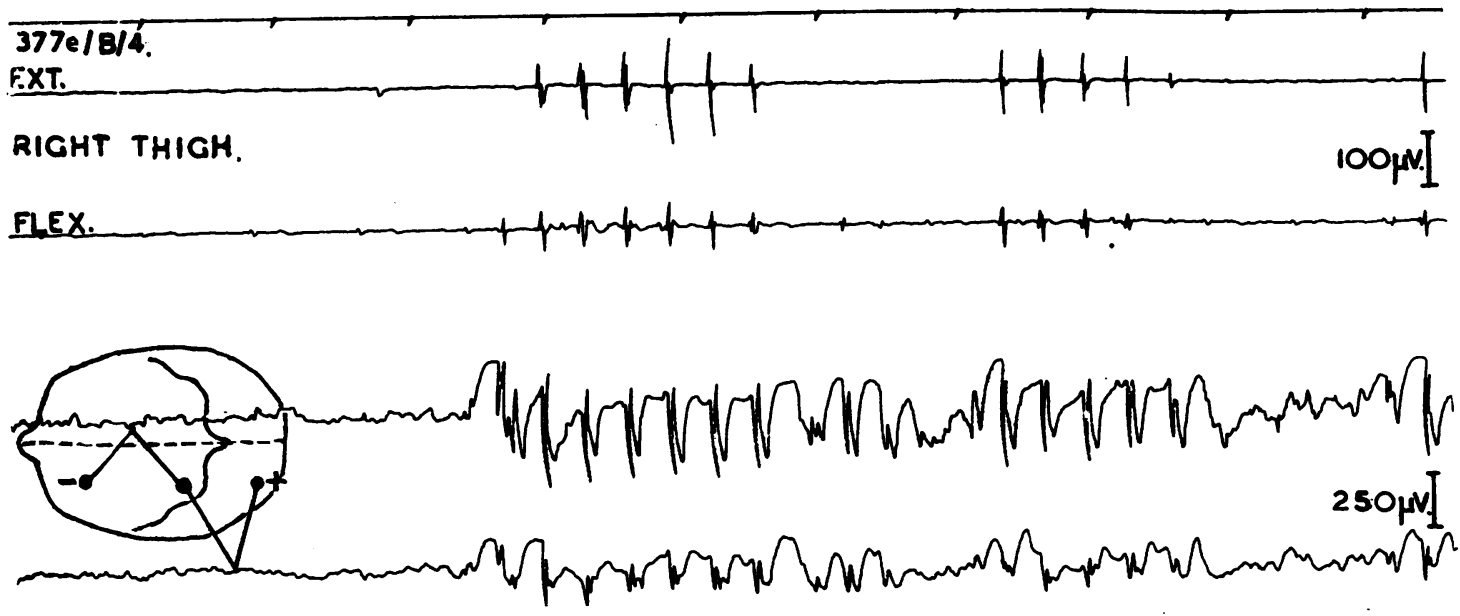

FIG. 14.- Record of the muscular jerkings accompanying wave and spike discharge evoked by overbreathing. Compare the small size of the muscle action potentials with those in the severe myoclonic jerkings (Fig. 4 (a)). The small deflections in the flexor record before and after the seizure are an artefact due to the electrocardiogram. 
record shown in Fig. $13(b)$. Here the sole discharge in the extensors takes place after three waves and spikes have occurred with an interval between them 20 per cent. shorter than that between those preceding them. Thus the differences in the two conditions may be due solely to the different amounts of facilitation occurring, and it is not necessary to postulate that the efferent cerebral discharges in them are different in either nature or magnitude.

In the periods following spontaneous seizures, overbreathing may sometimes evoke wave and spike discharges. In the later parts of the interseizure period these evoked outbursts are associated with muscular jerkings similar to those occurring in the spontaneous attacks, and the muscle action potentials are synchronous with the spikes in the EEG (Fig. 14), and here as in the myoclonic prodrome the discharge begins in the flexor muscles. In the earlier part of the interseizure period the number of wave and spike complexes evoked by a given amount of overbreathing is less than at a later time. Often only a single wave and spike or slow wave occurs (Fig. 15 (a)) and no muscle discharge is seen. If the muscle or group of muscles being tested is now stretched slightly it is often possible to pick up a simple rhythmic discharge from what is apparently a single motor unit. Under these conditions when a single wave and spike or slow wave occurs in the EEG the rhythm of the single unit is interrupted and a discharge is interpolated (Fig. $15(b)$ and (c)). In the latter part of the figure it seems that the rhythm is " reset " from the point where the interpolated discharge occurs. In this case the delay between the wave in the EEG and the muscle discharge is longer and less constant than it is when the regular repetitive spikes occur in the EEG. Probably the delay depends on the level of excitation at the motor neurone level at the time the efferent cerebral discharge reaches it.

\section{Discussion}

The results of the investigations described above in the patients with severe myoclonic jerkings confirm the findings of Grinker et al. (1938), and in the patient with wave and spike discharge with myoclonus those of Jasper and Andrews, (1938). The present cases of severe myoclonic jerkings show spikelike discharges in the EEG apparently exactly comparable with those described by Grinker et al. After discussing their findings Grinker et al. decided that the disturbances in myoclonic epilepsy probably had a cortical rather than a subcortical origin. The evidence available from the present studies does not make it profitable to speculate further on this point, for the disturbances in the EEG in myoclonic epilepsy are so large and so widespread that it seems highly unlikely that of cortex or basal structures, pyramidal or other efferent systems, any one will be active in isolation. In spite of the probable diffuse nature of the disturbances during the seizure there are indications for further study.
There are clearly changes in the tendon reflexes which indicate an increase in spinal excitability, at any rate for extensor reflex mechanisms. Since these changes are unaccompanied by any obvious changes in the EEG, some steadily maintained discharge affecting the spinal centres, or the removal of such a discharge, must be of importance in the periods between jerkings preceding the major convulsion. How far these changes in spinal excitability determine the pattern of the jerkings when these do occur is not clear, though it is obvious that the part played by them is considerable. The greater accessibility of the flexor mechanisms to the abnormal discharges in the two cases described, shown by the muscle action potentials, may be taken to suggest that although the pyramidal system is almost certainly not the only one discharging abnormally in these cases, it has the dominating influence at the spinal level.

It must be emphasized that the finding that muscular jerkings accompany spike discharges in the EEG does not apply to all types of myoclonic epilepsy. Many of the minor myoclonic phenomena that occur so frequently in epilepsy (Russell Reynolds (1861) has estimated that 75 per cent. ir of all epileptics have them in some form) seem 을 to show no correlates in the EEG. One subject at The David Lewis Colony showed a rhythmic twitching of the face, arm, and leg of one side the body only, which could not be associated wits any disturbance in the EEG. Unfortunately the jerkings in this patient ceased before full investigą tions could be made.

The findings in the patient with wave and spike discharge with mild mycolonus show a rather closer correlation of the spike of the complex with the burst of muscle action potentials than in similar cases described by Jasper. Like his cases, this patient also shows that the spike of the cerebral discharge and the muscle discharge may not be directly related but are probably both correlates of some third process. It seems that the presence of efferent cerebral discharges, in themselves subliminal for excitation at the spinal level, may be inferred from their effects on a purely spinal reflex mechanism, such as the rhythmic discharge of a stretched single motor unit. This suggests that if studies are carried out at the spinal reflex level, in conditions other than epilepsy, a closer correlation may be found between cerebral rhythms and peripheral effects than has so far been shown by studies at the lower level of the muscles themselves.

\section{Summary}

1. The EEGs of two subjects with severe myoclonic seizures and one subject with mild myoclonic jerkings accompanying wave and spike discharge in the EEG are described.

2. Repeated simultaneous recordings of the EEGs and muscle action potentials have been made in these subjects during myoclonic episodes.

3. All subjects show some form of spike discharge, with or without a slow wave, in the 

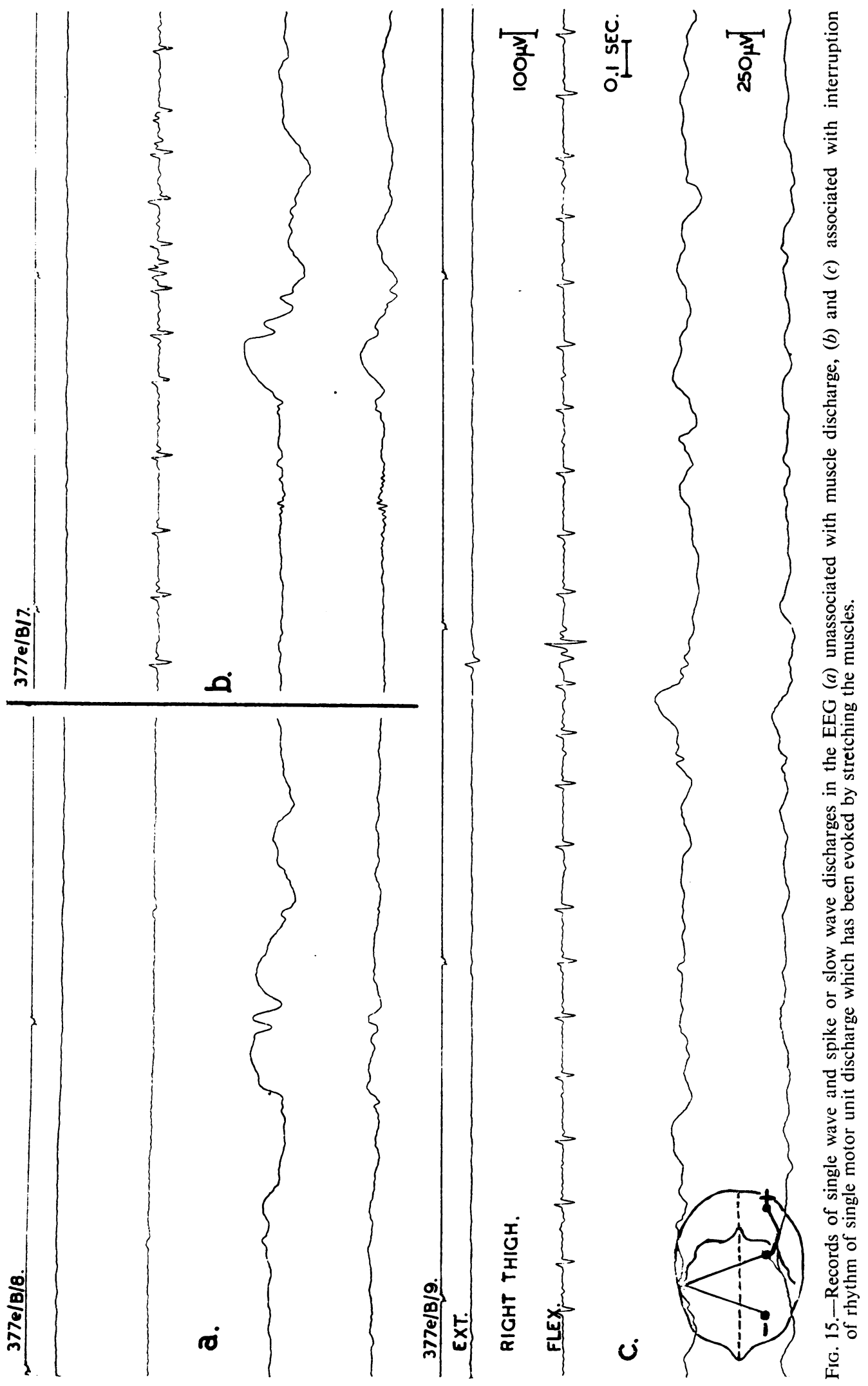

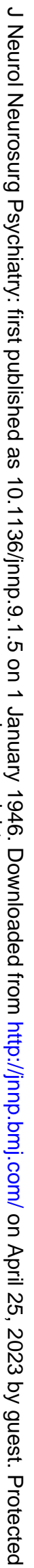


inter-seizure periods. In the seizures the patients with the severe myoclonic jerkings show discharges of spikes in their EEGs with a period of 30 to 75 msec. at a rate of 8 to 13 per second.

4. There is an accurate correlation between the occurrence of the spikelike discharges in the EEG and the action potentials of the muscular jerkings in the severe cases and a less accurate correspondence in the mild case.

5. The muscle action potentials show recruitment and facilitation having a different time course of decay for flexor and extensor muscles.

6 . In the periods between the spontaneous jerkings changes in spinal reflex excitability. may be demonstrated.

7. Important differences in the characters of the muscular jerkings in the severe myoclonic states and the milder state accompanying wave and spike discharge are described.

8. These findings are discussed and their bearing on the origin and mediation of the abnormal discharges in these conditions are considered.

The author would like to express his thanks to the Committee of The David Lewis Colony who made this work possible, to the Medical Research Council for facilities to complete it, and to Dr. Handley, Director of the Colony, for his advice and constant encouragement.

\section{REFERENCES}

Adrian, E. D., and Bronk, D. W. (1929). J. Physiol., $67,119$.

Buchthal, F., and Nielsen,'J. O. (1936). Skand. Arch. Physiol., 74, 202.

Gibbs, F. A., Davis, H., and Lennox, W. G. (1935). Arch. Neurol. Psychiat. Chicago, 34, 1133.

-, Gibbs, E. L., and Lennox, W. G. (1937). Brain, $60,377$.

- and Gibbs, E. L. (1941). Atlas of Electroencephalography, Cambridge, Mass.

Grinker, R., Serota, H., and Stein, S. (1938). Arch. Neurol. Psychiat. Chicago, 40, 968.

Jasper, H. H., and Andrews, H. L. (1938). J. Neurophysiol., 1, 87.

(1941). In Epilepsy and Cerebral Localisation, Penfield and Erickson, Ballière, Tyndall \& Cox, Loridon.

Offner, F. (1937). Rev. Sci. Instr., 8, 20.

Reynolds, Russell (1861). Epilepsy, Churchill, London Strauss, H., and Landis, C. (1938). Proc. Soc. exp. Biol. N.Y., 38, 369.

Toennies, J. F. (1938). Rev. Sci. Instr., 9, 95.

Walter, W. G. (1936). Lancet, 231, 305. 